\title{
Prebiotics Supplementation Impact on the Reinforcing and Motivational Aspect of Feeding
}

\author{
Anne-Sophie Delbès', Julien Castel', Raphaël G. P. Denis', Chloé Morel', \\ Mar Quiñones' ${ }^{1}$, Amandine Everard ${ }^{2}$, Patrice D. Cani ${ }^{2}$, Florence Massiera ${ }^{3}$ \\ and Serge $H$. Luquet ${ }^{1 *}$
}

1 Université Paris Diderot, Sorbonne Paris Cité, Unité de Biologie Fonctionnelle et Adaptative, CNRS UMR 8251, Paris, France, ${ }^{2}$ Walloon Excellence in Life Sciences and Biotechnology (WELBIO), Metabolism and Nutrition Research Group, Louvain Drug Research Institute, Université catholique de Louvain, Brussels, Belgium, ${ }^{3}$ Laboratoire de Recherche Nutritionnelle KOT CEPRODI SA, Paris, France

OPEN ACCESS

Edited by:

Julie A. Chowen,

Hospital Infantil Universitario

Niño Jesús, Spain

Reviewed by:

Daniela Cota

Institut National de la Santé et de la Recherche Médicale

(INSERM), France

Denis Richard,

Laval University, Canada

*Correspondence:

Serge H. Luquet serge.luquet@univ-paris-diderot.fr

Specialty section:

This article was submitted to Neuroendocrine Science, a section of the journal Frontiers in Endocrinology

Received: 15 February 2018 Accepted: 09 May 2018

Published: 29 May 2018

Citation:

Delbès A-S, Castel J, Denis RGP, Morel C, Quiñones M, Everard A, Cani PD, Massiera F and Luquet SH (2018) Prebiotics Supplementation Impact on the Reinforcing and Motivational Aspect of Feeding. Front. Endocrinol. 9:273. doi: 10.3389/fendo.2018.00273
Energy homeostasis is tightly regulated by the central nervous system which responds to nervous and circulating inputs to adapt food intake and energy expenditure. However, the rewarding and motivational aspect of food is tightly dependent of dopamine (DA) release in mesocorticolimbic (MCL) system and could be operant in uncontrolled caloric intake and obesity. Accumulating evidence indicate that manipulating the microbiotagut-brain axis through prebiotic supplementation can have beneficial impact of the host appetite and body weight. However, the consequences of manipulating the implication of the microbiota-gut-brain axis in the control motivational and hedonic/reinforcing aspects of food are still underexplored. In this study, we investigate whether and how dietary prebiotic fructo-oligosaccharides (FOS) could oppose, or revert, the change in hedonic and homeostatic control of feeding occurring after a 2-months exposure to high-fat high-sugar (HFHS) diet. The reinforcing and motivational components of food reward were assessed using a two-food choice paradigm and a food operant behavioral test in mice exposed to FOS either during or after HFHS exposure. We also performed mRNA expression analysis for key genes involved in limbic and hypothalamic control of feeding. We show in a preventive-like approach, FOS addition of HFHS diet had beneficial impact of hypothalamic neuropeptides, and decreased the operant performance for food but only after an overnight fast while it did not prevent the imbalance in mesolimbic markers for DA signaling induced by palatable diet exposure nor the spontaneous tropism for palatable food when given the choice. However, when FOS was added to control diet after chronic HFHS exposure, although it did not significantly alter body weight loss, it greatly decreased palatable food tropism and consumption and was associated with normalization of MCL markers for DA signaling. We conclude that the nature of the diet (regular chow or HFHS) as well as the timing at which prebiotic supplementation is introduced (preventive or curative) greatly influence the efficacy of the gut-microbiota-brain axis. This crosstalk selectively alters the hedonic or motivational drive to eat and triggers molecular changes in neural substrates involved in the homeostatic and non-homeostatic control of body weight.

Keywords: food intake, hedonic and motivational component, dopaminergic system, prebiotic, reward 


\section{INTRODUCTION}

Obesity and corollary pathologies, such as dyslipidemia, diabetes, and cardiovascular diseases are spreading in both developed and developing countries as a result of increased accessibility to energy-dense food associated with a general decrease in physical activity and energy expenditure (1). Whereas some genetic loci were clearly identified and extensively studied as monogenic causes for obesity, it is widely accepted that the metabolic syndrome is in essence a multifactorial disease that encloses a complex network of molecular, cellular, and physiologic alterations $(1,2)$. Understanding the complex pathology of the metabolic syndrome will be critical in shaping effective preventive and therapeutic strategies. However, despite the encouraging results obtained through pharmacological and surgical interventions, no effective anti-obesity treatment with long-lasting effects on body weight is nowadays available.

Proper energy balance is insured by the ability of the central nervous system to integrate nervous and circulating signals that reflect nutritional status to produce adaptive metabolic and behavioral responses aiming at maintaining body weight within a physiological narrow range (3). In addition, the rewarding aspects of energy-dense food largely depends on dopamine (DA) release from dopaminergic neurons in the ventral tegmental area that project to limbic regions, notably the prefrontal cortex and the nucleus accumbens $(\mathrm{NAcc})(4,5)$. This neural substrate referred as to mesolimbic "reward" circuit is instrumental in the encoding of both the volume of desire, i.e., "motivation" and the hedonic aspect, i.e., "liking" in food rewards (6).

Hence, the complex behavioral sequence leading to food intake results from the integration of metabolic needs but also reinforcing aspects of food. Multiple lines of evidence suggest that high-fat feeding and obesity per se can promote long-lasting adaptations in both hypothalamic and limbic regions thus leading to increased vulnerability to over-consume energy-dense food. In turn, such vulnerability can promote aberrant behaviors $(7,8)$ in which the reward becomes the primary driving force to consume energy-dense food (9-12). For instance, emerging theories suggest that chronic exposure to palatable food might impair the proper encoding of reward and, similar to drug of abuse, lead to desensitization of the DA mesolimbic system, and ultimately promote craving and addictive-like consummatory behavior $(4,6)$.

However, despite similar exposure to palatable and hypercaloric food, the development of eating-habits dissociated from actual homeostatic needs does not occur in every individual, suggesting differential degrees of vulnerability. In that regard, the microbiota-gut-brain axis has recently emerged as a key regulator of brain structures involved in stress-like responses $(13,14)$ together with resilience to high-fat-induced body weight gain (15). Dietary prebiotic such as the soluble fibers fructooligosaccharides (FOS) represents selectively fermented compounds that promote changes in the activity and composition of the gut microbiota, that are associated with a wide spectrum of beneficial effects including reduced appetite (16-20), decreased body weight (15), improved glucose metabolism (15), dampened susceptibility to stress $(21,22)$, and improved learning discrimination in rodents (23).

These observations suggest that prebiotic manipulation of the microbiota-gut-brain axis could directly impact both homeostatic and non-homeostatic control of food intake. However, the behavioral and molecular consequences of prebiotic supplementation onto the reinforcing and motivational components of food seeking have hitherto been largely unexplored.

In the current study, we investigated how administration of FOS could oppose-in a preventive-like approach or reverse-in a curative approach the behavioral and molecular adaptations induced by high-fat high-sucrose exposure and their consequences on food preference and motivation for food seeking.

\section{MATERIALS AND METHODS}

\section{Animals and Diets}

Ten-weeks-old male mice C57Bl/6J (25-30 g, Janvier, Le Genest Saint Isle, France) were housed in stainless steel cages in a room maintained at $22 \pm 1^{\circ} \mathrm{C}$ with light from 7:00 a.m. to 7:00 p.m. Food (Safe, Augy, France) and water were given ad libitum unless otherwise stated. C57Bl/6J were split in six groups $(n=12$ / group). The first four groups were exposed, respectively, during 2 -months to a control diet (Ctrl, 3,438 kcal/kg, protein $19 \%$, lipid 5\%, carbohydrates 55\%, reference \#U8959 version 63 Safe, Augy, France), a control diet enriched in fructo-oligosaccharide (Ctrl-FOS, $3,438 \mathrm{kcal} / \mathrm{kg}$, protein $17 \%$, lipid $8 \%$, carbohydrates $49 \%$, oligofructose $10 \%$ ), a high-fat high-sugar diet (HFHS, $4,362 \mathrm{kcal} / \mathrm{kg}$, proteins $20 \%$, lipid $23 \%$, carbohydrate $37 \%$, reference \#U8954 version 14 Safe, Augy, France), and an HFHS diet enriched in fructo-oligosaccharide (HFHS-FOS, 4,362 kcal/kg, proteins $18 \%$, lipid $20 \%$, carbohydrate $34 \%$, oligofructose $10 \%$ ). The last two groups were subjected to a 2-months HFHS diet and split in two groups $(n=12)$ that received during the following 2-months a "control" diet (HFHS/Ctrl) and a control diet enriched in fructo-oligosaccharide (HFHS/Ctrl-FOS). Groups supplemented with FOS will also be referred in the text by preventive (Ctrl-FOS, HFHS-FOS) or corrective (HFHS/Ctrl-FOS) effects of FOS. All animal experiments were performed with approval of the Animal Care Committee of the University Paris Diderot-Paris 7 and according to European directives.

\section{Body Composition Analysis}

Mice were monitored for body weight and composition at the beginning and the end of the experiment. Body mass composition (lean tissue mass, fat mass, free water, and total water content) was analyzed using an Echo Medical systems' EchoMRI (Whole Body Composition Analyzers, EchoMRI, Houston, TX, USA), according to manufacturer's instructions.

\section{Measurement of Food Intake and Food Preference}

Analyses were performed in an automated online measurement system using high sensitivity feeding and drinking sensors and an infrared beam-based activity monitoring system (Phenomaster, TSE Systems GmbH, Bad Homburg, Germany). 
Mice were evaluated for food preference when exposed to HFHS and control chow diet (CTRL). Food preference was measured during six short sessions of $2 \mathrm{~h}$ (days 1-6) using animals as their own controls.

\section{Operant Conditioning System}

Operant responding performance was performed as previously described (24). Computer-controlled operant conditioning was conducted in 12 identical conditioning chambers equipped with a swiveling infusion device (Phenomaster, TSE Systems $\mathrm{GmbH}$, Bad Homburg, Germany). Each chamber contains an operant wall with a food cup, two levers located $3 \mathrm{~cm}$ lateral to the food cup, with the left lever designated the active lever (for food pellet delivery). Mice are maintained at 90\% of initial body weight to facilitate initial learning and performance of a fixed ratio (FR1) operant learning task. The reinforcer was a single 20-mg peanut butter flavored sucrose tablet (TestDiet, Richmond, VA, USA).

Operant training was carried out over six consecutive days with two overnight fix ratio of 1 (FR) and then four consecutive days with one 2-h trial of FR1 per day. At the conclusion of the 6-days operant training period, animals were given four trials to lever press for sucrose under a progressive ratio of 3 (PR), lever press requirement for each subsequent reinforcer increased by 3 with an initial requirement of 3 lever press $(r=3 N+3 ; N=$ reinforcer number).

The PR schedule requires the mouse to perform an increasing number of lever presses for each consecutive reward, the number of rewards received (also called breakpoint) was used to assess motivation or effort to work for a food reward.

At the end of the experiment, animals were sacrificed, brain, liver, cecum tissues, and plasma collected.

\section{Gut Microbiota Analysis}

At the end of the experiment, the total cecum content was collected and weighed before storage at $-80^{\circ} \mathrm{C}$. Metagenomic DNA was extracted from the cecal content using the QIAamp DNA stool mini kit (Qiagen, Hilden, Germany) according to the manufacturer's instructions. Quantitative PCR (qPCR) for total bacteria, Bifidobacterium spp., Lactobacillus spp., Akkermansia muciniphila, Roseburia spp., and Bacteroides-Prevotella spp. were performed by using Mesa Fast qPCR ${ }^{\mathrm{TM}}$ (Eurogentec, Seraing, Belgium). Real-time PCRs were performed with the StepOnePlus ${ }^{\mathrm{TM}}$ realtime PCR system and software (Applied Biosystems, Den Ijssel, The Netherlands). The primers sequences were described previously $(15,25)$. Cycle threshold of each sample was then compared with a standard curve (performed in triplicate) made by diluting genomic DNA obtained from BCCM/LMG (Ghent, Belgium) or DSMZ (Braunshweig, Germany). Prior to isolating the DNA, the cell counts were determined by BCCM/LMG or DSMZ, respectively; fivefold serial dilution of Bifidobacterium animalis BCCM/ LMG 18900 for Bifidobacterium spp., Bacteroides fragilis BCCM/ LMG 10263 for Bacteroides-Prevotella spp., Lactobacillus acidophilus DSM 20079 for Lactobacillus spp., A. muciniphila MucT (ATTC BAA-835, DSMZ22959) for A. muciniphila, Roseburia intestinalis (DSMZ 14610) for Roseburia spp., and Lactobacillus acidophilus DSM 20079 for total bacteria.

\section{Isolation of Total RNA and Quantitative RT-PCR}

From all groups, total hypothalamic RNA was extracted and analyzed by qRT-PCR for agouti-related protein (AgRP), neuropeptide Y (NPY), pro-opiomelanocortin (POMC), and cocaine and amphetamine-regulated transcript (CART), and total nucleus accumbens (NAcc) RNA was extracted and analyzed by qRT-PCR for dopamine transporter (DAT), dopamine receptor D1 (DR1), dopamine receptor D2 (DR2), dopamine beta hydroxylase (DBH), and tyrosine hydroxylase (TH).

Total RNA was isolated as described previously (9). We retro transcribed $1 \mu \mathrm{g}$ RNA using Superscript II (Invitrogen). Realtime quantitative (qRT-PCR) analyses were performed with $25 \mathrm{ng}$ cDNA and $250 \mathrm{nM}$ sense and antisense primers (Eurogentec) in a final reaction volume of $25 \mu$ l by using qPCR Core Kit (Eurogentec) and the MyiQ real-time PCR detection system (Bio-Rad). Specific primers were designed using Primer Express software (version 1.0, Applied Biosystems) and primers sequences and housekeeping gene (HKG) are listed below. Relative quantification of hypothalamic and NAcc RNA for each gene was calculated after normalization to HKG by using the comparative $\mathrm{Ct}$ method.

\begin{tabular}{|c|c|}
\hline Primers & Sequences \\
\hline POMC & $\begin{array}{l}\text { Forward: 5'-AGTGCCAGGACCTCACCA-3' } \\
\text { Reverse: 5'-CAGCGAGAGGTCGAGTाTG-3' }\end{array}$ \\
\hline NPY & $\begin{array}{l}\text { Forward: 5'-CCGCTCTGCGACACTACAT-3' } \\
\text { Reverse: 5'-TGTCTCAGGGCTGGATCTCT-3' }\end{array}$ \\
\hline $\mathrm{AgRP}$ & $\begin{array}{l}\text { Forward: 5'-CGGAGGTGCTAGATCCACAGA-3' } \\
\text { Reverse: 5'-AGGACTCGTGCAGCCTTACAC-3' }\end{array}$ \\
\hline CART & $\begin{array}{l}\text { Forward: 5'-CGAGAAGAAGTACGGCCAAG-3' } \\
\text { Reverse: 5'-CTGGCCCCTITCCTCACT-3' }\end{array}$ \\
\hline DAT & $\begin{array}{l}\text { Forward: 5'-GCCCTACCTGCTCTTCATGC-3' } \\
\text { Reverse: 5'-GGATGACAGTGAAGCCCACA-3' }\end{array}$ \\
\hline DR1 & $\begin{array}{l}\text { Forward: 5'-TCTGGTITACCTGATCCCTCA-3' } \\
\text { Reverse: 5'-GCCTCCTCCCTCTTCAGGT-3' }\end{array}$ \\
\hline DR2 & $\begin{array}{l}\text { forward: 5'-TGAACAGGCGGAGAATGG-3' } \\
\text { Reverse: 5'-CTGGTGCTTGACAGCATCTC-3' }\end{array}$ \\
\hline $\mathrm{DBH}$ & $\begin{array}{l}\text { Forward: 5'-ATCTCCATGCATTGCAACAA-3' } \\
\text { Reverse: 5'-AGGCTGCAGATTCCACTCAC-3' }\end{array}$ \\
\hline $\mathrm{TH}$ & $\begin{array}{l}\text { Forward: 5'-GGTATACGCCACGCTGAAGG-3' } \\
\text { Reverse: 5'-TAGCCACAGTACCGTTCCAGA-3' }\end{array}$ \\
\hline RPL19 HKG & $\begin{array}{l}\text { Forward: 5'-GGGCAGGCATATGGGCATA-3' } \\
\text { Reverse: 5'-GGCGGTCAATCTTCTTGGATT-3' }\end{array}$ \\
\hline H1A HKG & $\begin{array}{l}\text { Forward: 5'-AGAAGAACAACAGCCGCATC-3' } \\
\text { Reverse: 5'-TGCACCAGTGTGCCTTATT-3' }\end{array}$ \\
\hline HPRT HKG & $\begin{array}{l}\text { Forward: 5'-GTTGGATACAGGCCAGACTTTGTTG-3' } \\
\text { Reverse: 5'-GATTCAACTTGCGCTCATCTTAGGC-3' }\end{array}$ \\
\hline CYCLOA HKG & $\begin{array}{l}\text { Forward: 5'-ACGCCACTGTCGCTIITC-3' } \\
\text { Reverse: 5'-GCAAACAGCTCGAAGGAGAC-3' }\end{array}$ \\
\hline
\end{tabular}

\section{Statistical Analysis}

Displayed values are mean \pm SEM. Variance equality was analyzed by a paired $t$-test (GraphPad Prism $\left.6^{\circledR}\right)$. Unless otherwise stated, comparisons between groups were carried out using analysis of variance (ANOVA, GraphPad Prism 6 ${ }^{\circledR}$ ). A $P$-value of less than 0.05 was considered statistically significant. 


\section{RESULTS}

\section{Preventive vs Corrective Prebiotic Supplementation Differentially Impact Body Weight and Gut Microbiota Composition}

Four groups were subjected to a 2-months long preventing-like approach of prebiotic supplementation in which two groups received regular chow diet with or without soluble fibers FOS (Ctrl or Ctrl-FOS groups) while two last groups received a similar treatment but were raised on palatable high-fat highsucrose diet with or without FOS (HFHS or HFHS-FOS groups) (Figure 1A). An additional two animal cohorts were dedicated to explore the corrective potency of prebiotic supplementation onto metabolic and behavioral changes induced by a 2 months exposure to HFHS diet. In this setting, 12 weeks-old C57Bl/6J male mice were first raised on HFHS diet for 2-months then shifted onto Ctrl or Ctrl-FOS diet and will be referred as to HFHS/Ctrl or HFHS/Ctrl-FOS, respectively (Figure 1A). Based on the extensive literature in the field, we chose a $10 \%$ FOS enrichment since it was described to promote metabolic benefits (26-28) together with improvement in learning discrimination and improved cognitive performances $(23,29)$.

While average body weight did not differ among groups before the treatment, a significant increase in body weight was reached after HFHS but not HFHS-FOS exposure (Figures 1B,E,H). Body weight gain was mostly attributable to adipose tissue as revealed by body composition analysis (Figures 1C,D,F,G; Figures S1A-D in Supplementary Material). In HFHS/Ctrl and HFHS/Ctrl-FOS groups, the switch of HFHS to control diet stopped diet-induced body weight gain (Figure $\mathbf{1 H}$ ) in similar way between FOS and non-FOS-treated group (Figure 1H; Figure S1E in Supplementary Material).

Prebiotic supplementation is known to change the composition and activity of specific gastrointestinal microbiota $(30,31)$. Therefore, we decided to investigate if specific bacteria were modified following our diets. qPCR analysis of the cecum bacterial content revealed that the prevalence of Bifidobacterium spp., A. muciniphila, and to a lesser extent Lactobacillus spp. was significantly increased but only in condition in which both prebiotics and HFHS were combined either simultaneously or when FOS supplementation followed HFHS exposure. It is worth to mention that other bacterial families, such as Roseburia spp. and Bacteroides spp., were not affected by dietary regimens (Figures 1I,J). However, when mice were exposed to Ctrl or Ctrl-FOS diets, we could not identify significant changes in the microbiota ecosystem (Figures 1I,J).

\section{Timing in Prebiotic Supplementation Is Instrumental in the Beneficial Impact on Hedonic and Motivational Component Feeding}

Previous data suggest that modifications in the microbial diversity may influence food choice and tropism in the host and participate to satiety responses by regulating the gut-brain axis $(16,30,32)$.
After nutritional manipulation aimed at either preventing or correcting any metabolic and behavioral changes induced by HFHS exposure, the six groups were subjected to a two-food choice paradigm consisting in a seven daily consecutive $2 \mathrm{~h}$-limited access to both Ctrl and HFHS diet followed by an overnight exposure to food choice (Figure 2A). Short-term access to two-food choice aimed at deciphering the preference to select and consume palatable over chow pellets in a non-fasted condition. While Ctrl-fed animals displayed a tropism for HFHS over control chow diet (CTRL), Ctrl-FOS maximized their consumption of HFHS starting from the first session (Figures 2B-D). During the overnight exposure to food choice, a similar pattern was observed with increased HFHS consumption in Ctrl and Ctrl-FOS animals with an overall $90 \%$ preference for the palatable diet (Figure 2E).

Interestingly both cohorts exposed to HFHS diet (HFHS and HFHS-FOS) displayed minimal consumption of palatable diet when given the choice on a short period of time (Figures 2B-D), in agreement with the reduction in palatable food preference observed in animal fed with high-fat diet (33). This result contrasted, however, with a large preference for HFHS over CTRL in the overnight access in both HFHS and HFHS-FOS groups (Figure 2E). In addition, FOS supplementation in HFHS-FOS led to increased tropism for palatable diet compared to HFHS group (Figure 2E). These results indicate that mice exposed to diets supplemented with FOS (Ctrl or HFHS) show increased preference for palatable food (Figures 2B-E).

In sharp contrast with the lack of preventive action, when prebiotics were added in the diet after a 2 months HFHS exposure (HFHS/Ctrl-FOS), we observed a strong corrective action of FOS on both 2 -h time-restricted and overnight palatable diet intake (Figures 2F-I).

Food preference and seeking strongly rely on dopamine whose release within the mesocorticolimbic (MCL) system participate in driving the motivational and reinforcing values of food reward $(6,34-36)$. Hence, we next sought to behaviorally dissect the consequences of FOS supplementation on the motivational drive to obtain food rewards. After nutritional manipulation, the different cohorts underwent through an operant conditioning task to obtain food rewards. Animals were first subjected to fixed ratio (FR) reinforcement schedule in which a single lever press triggers the delivery of a palatable food pellet. Once mice have reached their discriminatory ability between active and inactive lever, they are shifted to a progressive ratio (PR) in which the number of lever presses required to obtain a reward increases progressively (Figure 3A). If a subject abnormally inflates the reinforcing aspect of a reward it will be more likely to exert effort to obtain it. Alternatively, if the perceived value of the reward is abnormally diminished, the willingness to engage in effortful behavior to obtain it will be reduced. In all conditions, operant responding for sucrose pellets reward was evaluated on mice gradually food restricted to $90 \%$ of body weight or following acute overnight in fed or fasting condition (Figure 3A).

Here again, the behavioral output of prebiotic supplementation was different according to the timing (preventive or corrective) of FOS addition in the diet. Ctrl and Ctrl-FOS groups had a similar profile with enhanced operant responding for food reward compared to both HFHS and HFHS-FOS group 


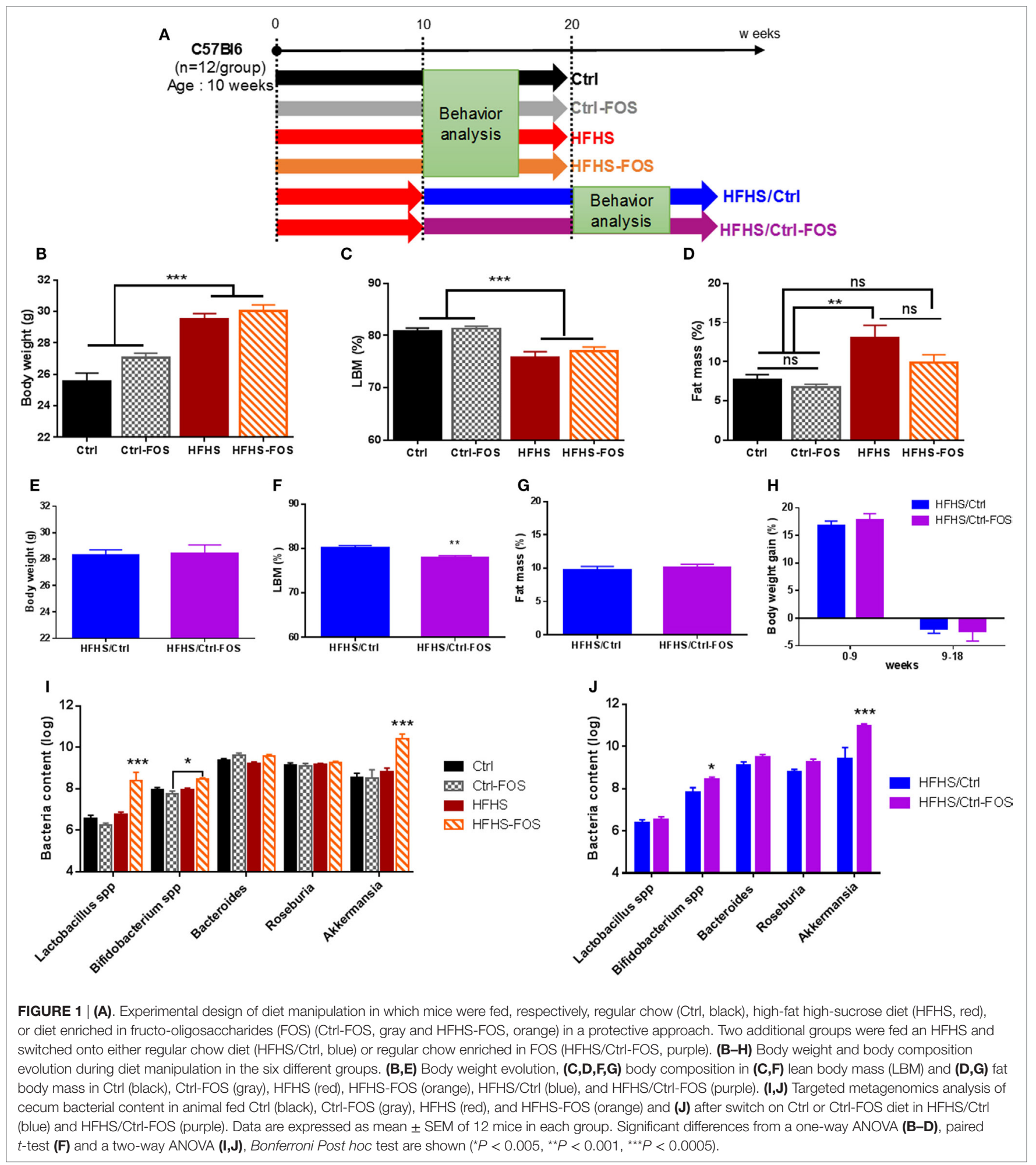

(Figure 3B). These results are consistent with the previous studies showing attenuated operant performance in high-fat fed animals (37). Surprisingly, FOS addition did not alter the number of collected rewards (Figure 3B), active lever press (Figure 3C), and discriminatory capacity between active and inactive lever
(Figures 3D,J) in animal exposed to Ctrl diet (Ctrl and Ctrl-FOS) under both chronic and acute fasting-induced body weight loss (Figures 3E,F,H). However, FOS supplementation on HFHS diet decreased the motivational drive to collect food reward under conditions of drastic energy deprivation (Figures 3G,I). 


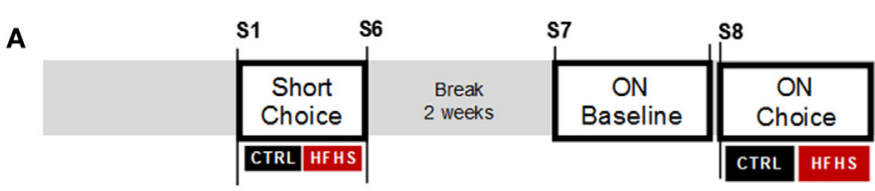

Short 8 shorts sessions of $2 \mathrm{~h}(3 \mathrm{pm}$ to $5 \mathrm{pm})$ ON: Overnight session (5pm to $10 \mathrm{pm})$
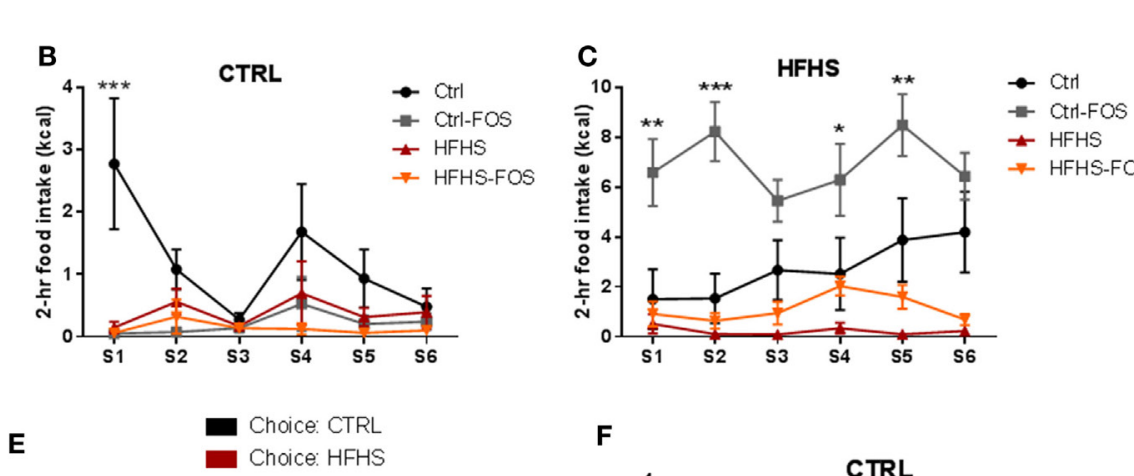

$\mathbf{F}$
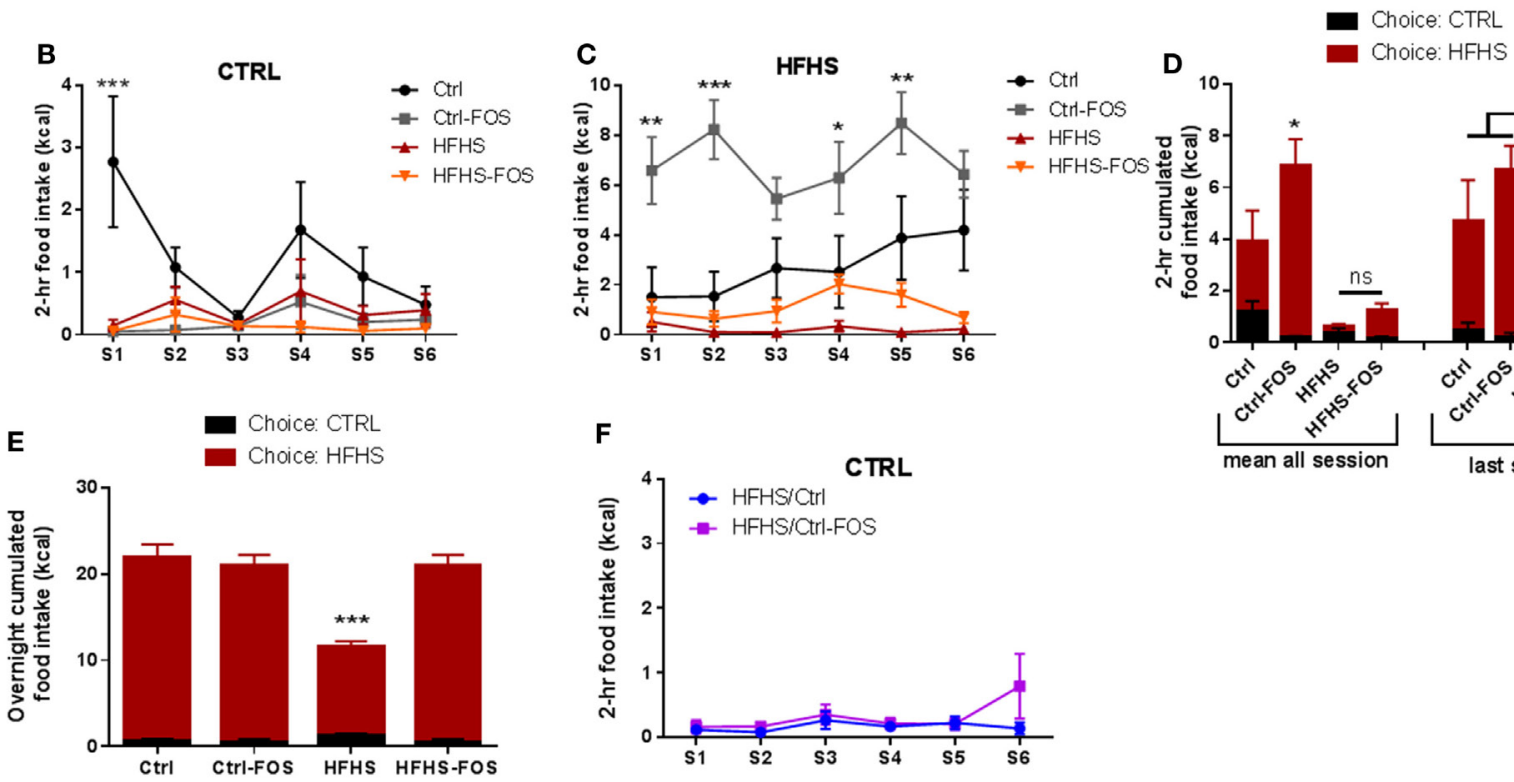

G

HFHS

H
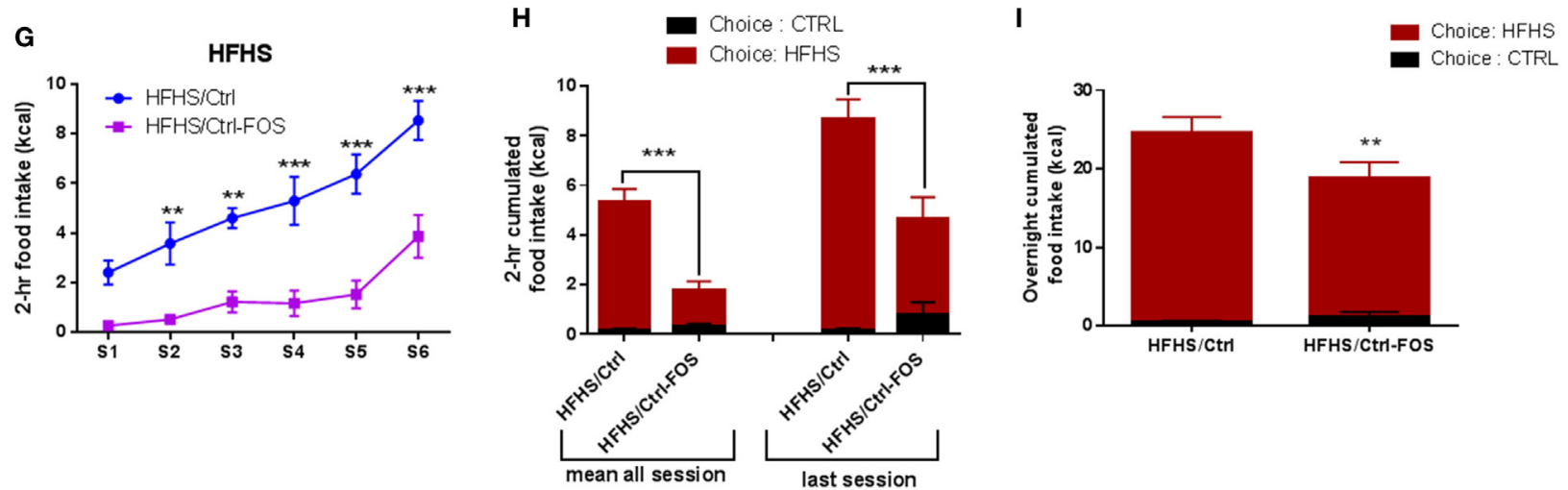

FIGURE 2 | (A) Experimental design for food tropism analysis using short or overnight access to a two-food choice protocol using regular chow diet or palatable HFHS diet for six mice per group. Regular chow diet [CTRL (B,D,F,H)] or HFHS intake (C,D, G,H) during seven sessions consisting of a 2-h two-food choice access, (D,H) representative tropism for CTRL vs HFHS as average cumulated intake through all session or during the last session, (E,I) representative tropism for CTRL vs HFHS as average cumulated intake overnight in Ctrl (black), Ctrl-fructo-oligosaccharides (FOS) (gray), HFHS (red), HFHS-FOS (orange), HFHS/Ctrl (blue), and HFHS/ Ctrl-FOS (purple). Data are expressed as mean \pm SEM of six mice in each group. Significant differences from a two-way ANOVA, Bonferroni Post hoc test $\left({ }^{\star} P<0.005,{ }^{\star \star} P<0.001,{ }^{\star \star \star} P<0.0005\right)$ (B-I).

A similar experimental design was carried out to evaluate operant conditioning in animals previously fed with an HFHS diet and shifted under Ctrl or Ctrl-FOS diets (HFHS/Crtl, HFHS/ Ctrl-FOS).

While a corrective property of FOS supplementation was evident in palatable diet intake and preference (Figures 2F-I), HFHS/Crtl and HFHS/Ctrl-FOS exhibited identical performance in every aspect of operant response for food reward in both tested conditions (chronic or acute food deprivation) (Figures 3K-Q).

These results highlight that prebiotics might exert a distinct and specific action onto the hedonic "liking," and motivational "wanting" drive to consume palatable food as well as feeding response to energy deprivation. Furthermore, our data support the notion that time-dependent exposure to prebiotics may be instrumental in their action onto reward-seeking behavior. 


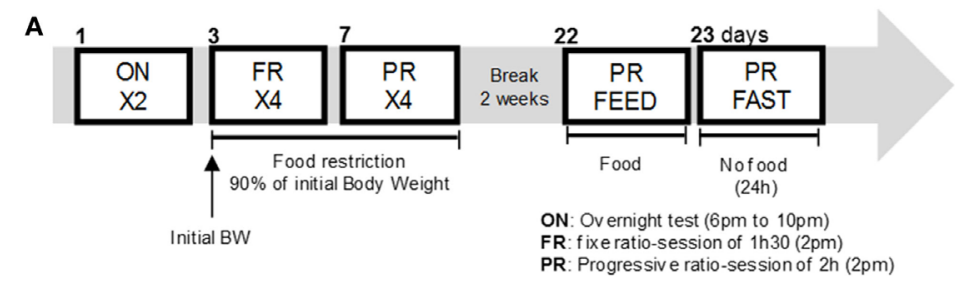

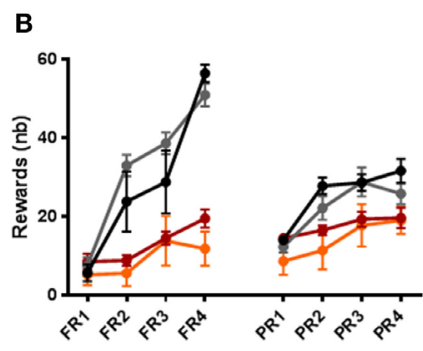
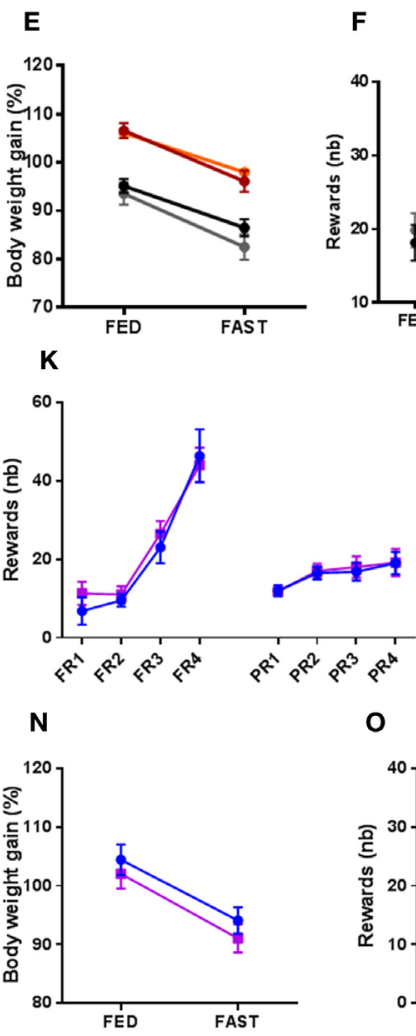

C

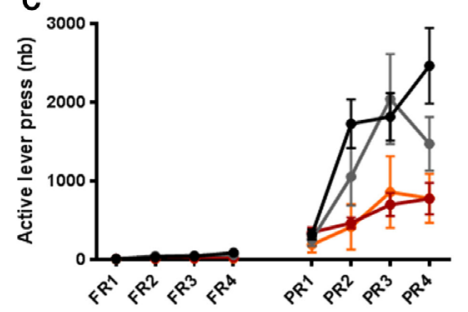

G
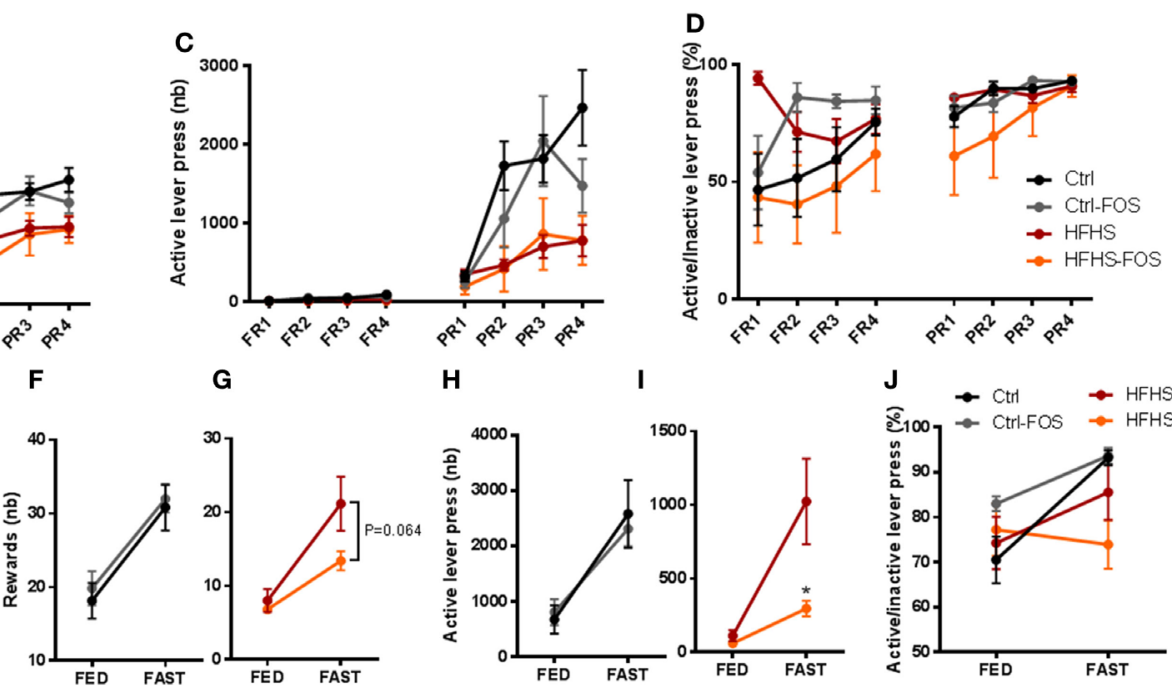

L
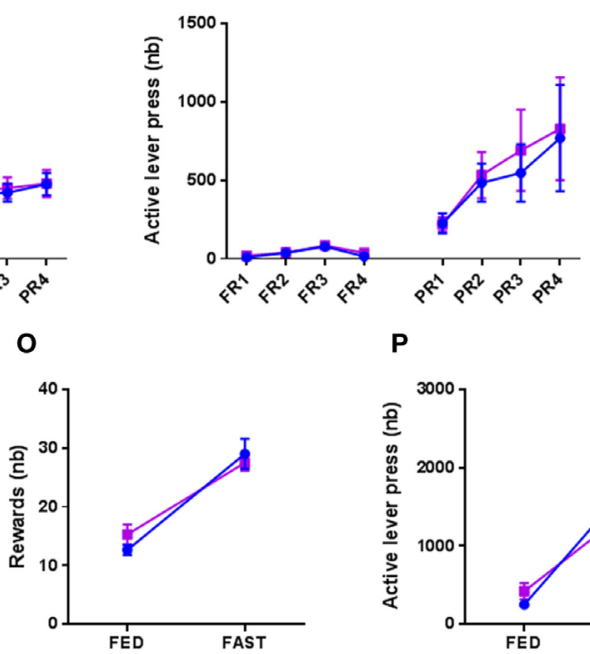

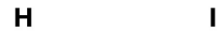

I
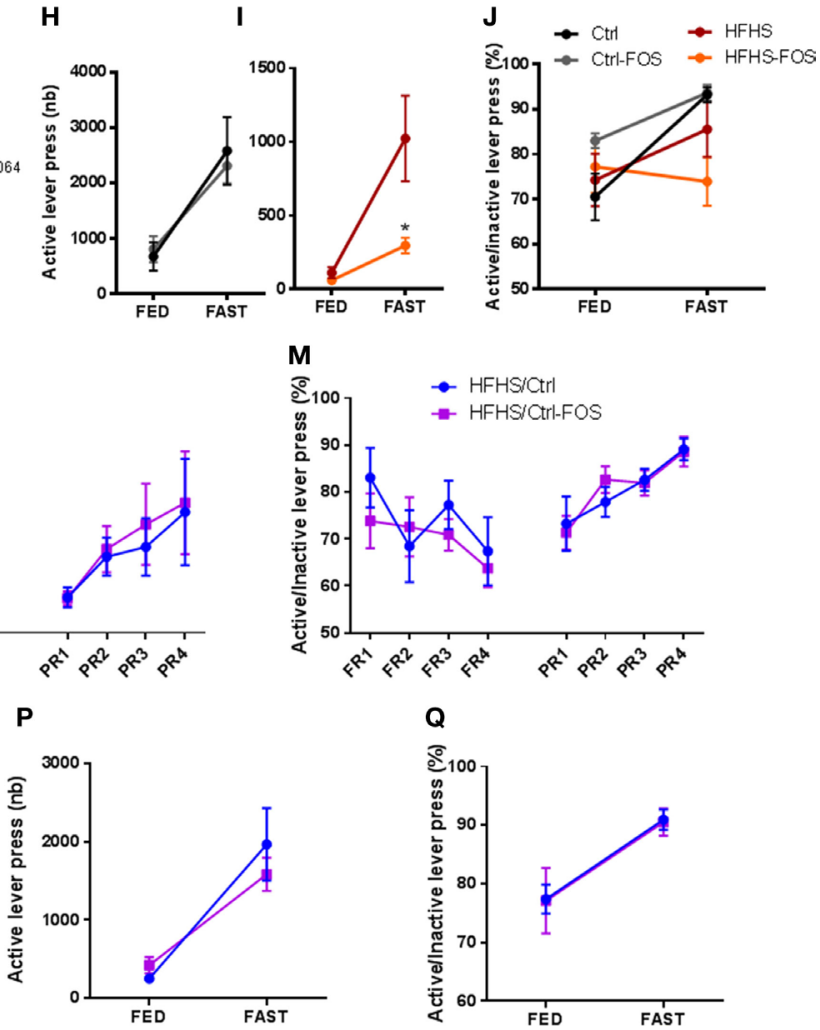

FIGURE 3 | (A) Experimental design for the operant responding performance assessment for six mice per group. (B,K) Reward number, (C,L) active lever press, $\mathbf{( D , M ) ~ r a t i o ~ b e t w e e n ~ t h e ~ a c t i v e ~ a n d ~ i n a c t i v e ~ l e v e r ~ p r e s s e s ~ i n ~ ( B - D ) ~ C t r l ~ ( b l a c k ) , ~ C t r l - f r u c t o - o l i g o s a c c h a r i d e s ~ ( F O S ) ~ ( g r a y ) , ~ H F H S ~ ( r e d ) , ~ ( K - M ) ~ H F H S - F O S ~ ( o r a n g e ) , ~}$ HFHS/Ctrl (blue), and HFHS/Ctrl-FOS (purple) during a 90\% body weight reduction. (E,N) body weight change, reward number (F,G,O), active lever press $\mathbf{( H , I , P ) ~ o r ~ a c t i v e ~ v s ~ i n a c t i v e ~ l e v e r ~ p r e s s ~ r a t i o ~ ( J , Q ) ~ i n ~ r e s p o n s e ~ t o ~ a n ~ o v e r n i g h t ~ f a s t ~ i n ~ C t r l ~ ( b l a c k ) , ~ C t r l - F O S ~ ( g r a y ) , ~ H F H S ~ ( r e d ) , ~ H F H S - F O S ~ ( o r a n g e ) , ~ H F H S / C t r l ~}$ (blue), and HFHS/Ctrl-FOS (purple). Data are expressed as mean \pm SEM of six mice per group. Significant differences from a two-way ANOVA, Bonferroni Post hoc test are shown $\left.{ }^{\star} P<0.005\right)$ (I).

\section{Timing in Prebiotic Supplementation Is Instrumental in Molecular Adaptation in Mesolimbic and Hypothalamic Structures}

The action of feeding results from the ability of the brain to properly integrate circulating signals of hunger and satiety together with food-related cues coding for palatable and rewarding values (38). The hypothalamus-brainstem axis, by primarily encoding metabolic needs, is regarded as the key neural network in the homeostatic control of body weight whereas the dopaminergic system is mainly involved in encoding the rewarding and reinforcing values of food seeking. Hence, hypothalamic-brainstem 
circuit is typically referred as to homeostatic while mesolimbic circuit are referred as to non-homeostatic regulation of feeding $(38,39)$. Interestingly, obesity and high-fat feeding have been shown to provoke various adaptive changes in both MCL and hypothalamic structures which could account for the toxic effect of energy-dense food $(8,10,40-43)$.

We therefore explored how time-dependent prebiotic manipulation modulated the molecular adaptations of dopaminoceptive and hypothalamic structures in response to high-fat feeding. mRNA were extracted from the NAcc and hypothalamus and analyzed for expression of genes involved in dopamine synthesis and signaling, i.e., DAT, DBH, DR1, DR2, and TH in the NAcc, while genes encoding neuropeptides involved in melanocortin signaling and body weight regulation i.e., NPY, agouti-related protein (AgRP), POMC, and cocaine and amphetamine-regulated transcript (CART) in the hypothalamus (Figures 4A,B).

In a preventive approach, the effect of FOS addition to Ctrl or HFHS diets was evident in hypothalamic expression of energy-related neuropeptides but failed to alter expression of dopamine-associated genes in the NAcc. Both Ctrl and Ctrl-FOS displayed similar levels of mRNA encoding DAT and DR2 that were significantly higher than those observed in either HFHS or HFHS-FOS (Figure 4A). In the hypothalamus, however, while exposure to energy-dense food led to increased mRNA contents for NPY and AgRP in HFHS group, prebiotic supplementation induced a significant decrease in NPY expression (Figure 4B). These results are in agreement with published observations showing that high-fat diet and/or obesity result in decreased expression of DR2 and DAT $(7,8)$ and, on the one hand, increased expression of hypothalamic orexigenic peptides $(44,45)$. Our results show that, in a preventive-like approach, FOS supplementation partially restores hypothalamic expression of orexigenic peptides but fails to correct the modifications induced by energy-dense food in the NAcc (Figures 4A,B).

Surprisingly, FOS enrichment had an opposite consequence onto hypothalamic peptides on mice exposed to Ctrl or HFHS

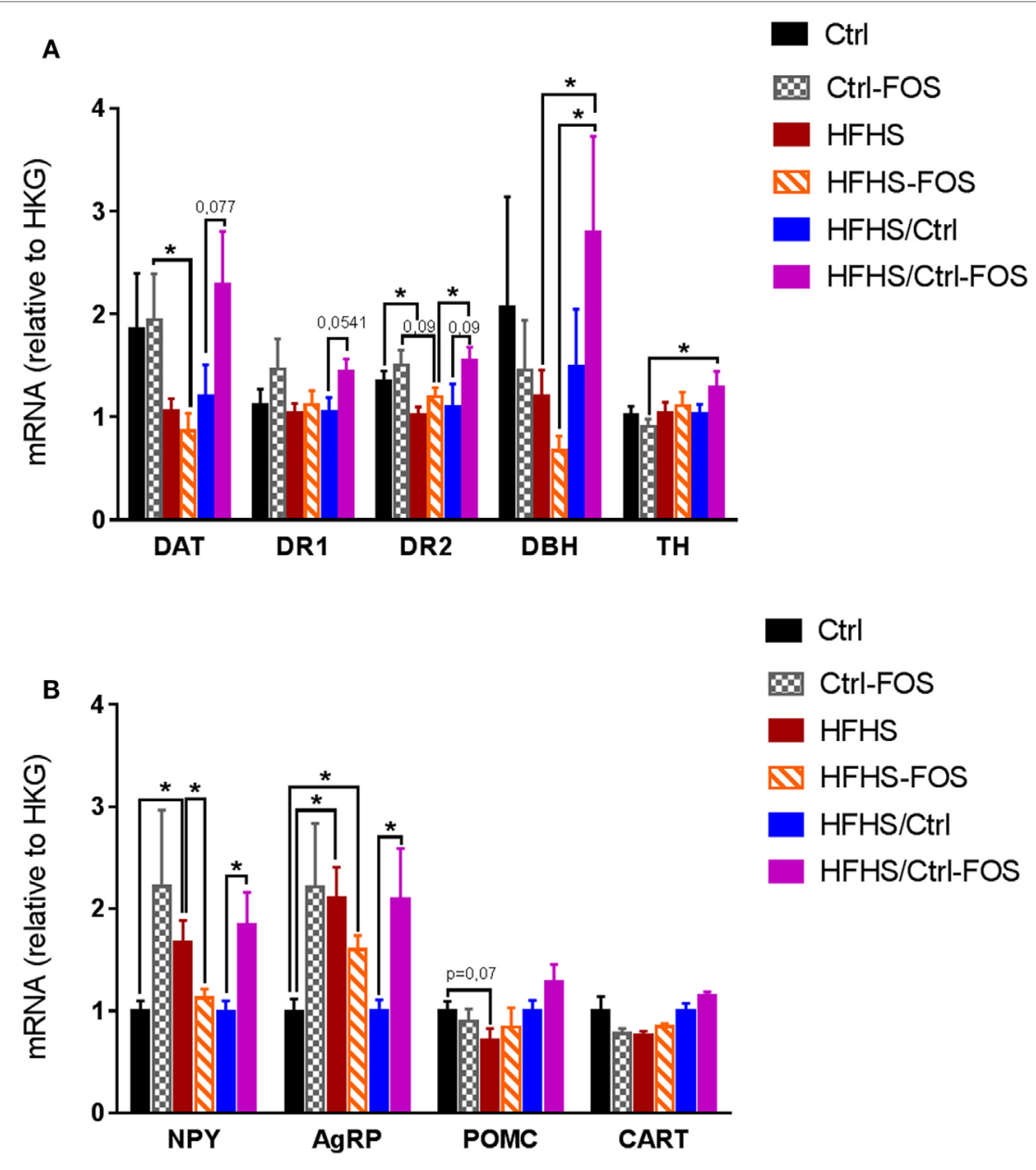

FIGURE 4 | Real-time PCR analysis of mRNA content (A) in the nucleus accumbens, for gene encoding for dopamine transporter (DAT), dopamine receptor 1 (DR1), dopamine receptor 2 (DR2), dopamine beta hydroxylase (DBH), and tyrosine hydroxylase (TH) and (B) in the hypothalamus for neuropeptide $\mathrm{Y}$ (NPY), Agouti-related protein (AgRP), Pro-opiomelanocortin (POMC), and cocaine and amphetamine-regulated transcript (CART) in Ctrl (black), Ctrl-fructo-oligosaccharides (FOS) (gray), HFHS (red), HFHS-FOS (orange), HFHS/Ctrl (blue), and HFHS/Ctrl-FOS (purple). Expression level corresponds to a ratio relative to housekeeping gene (HKG). Data are expressed as mean \pm SEM of eight mice in each group. Significant differences from a two-way ANOVA, Bonferroni Post hoc test $\left({ }^{*} P<0.005\right)(\mathbf{A}, \mathbf{B})$ 
diets. Ctrl-FOS diet led to increases in both orexigenic peptides NPY and AgRP compared to Ctrl group while the same nutritional manipulation operated onto HFHS diet led to a decrease of these peptides (Figure 4B). In both conditions, the modulation of NPY and AgRP were not counterbalanced by a change in the expression of the anorectic transcripts for POMC or CART (Figure 4B).

This result provides a molecular underpinning supporting the relative hyperphagia observed in Ctrl-FOS compared to Ctrl animals in food choice condition (Figures 2C,D). In the same food choice paradigm, FOS enrichment in HFHS diet led to increased consumption of palatable diet in an overnight session (Figure 2E) which point toward a decorrelation between hypothalamic decrease in NPY, AgRP and food reward seeking.

In a corrective-like approach, however, prebiotic supplementation to Ctrl diet in animal previously exposed to HFHS diet for 2 months fully restored Nacc level of DAT, DR2, and DBH (Figure 4A). However, while the shift onto Ctrl diet was per se sufficient to restore normal hypothalamic levels for NPY and AgRP, this benefic action was counterbalanced by FOS addition which was associated to sustained level of both orexigenic peptides (Figure 4B).

Altogether our results show that the molecular adaptations induced by high-fat feeding in brain structures that govern food intake in response to either metabolic demand or reward can be restored or opposed by prebiotic supplementation. Importantly, the timing in FOS supplementation together with the nature of the diet in which FOS is introduced with have critical impact on the direction by which prebiotic will operate the adaptive changes in MCL or hypothalamic structures and ultimately predict the ability of prebiotic to change food tropism and rewardseeking behavior.

\section{DISCUSSION}

While metabolic needs are primarily encoded in the hypothalamus, the reinforcing value of food encompasses a multisensory component including flavors and texture which ultimately modulates the release of DA in the MCL system. In modern society, calorie-dense foods are widely available and were associated with the progression of obesity together with the development of compulsive eating in which reward-driven eating behaviors are bypassing homeostatic regulation of nutrients intake $(4,46,47)$. The microbiota-gut-brain axis has emerged as a pivotal player in appetite control as well as reward-driven behavior (48).

In the present study, we described the impact of prebiotic supplementation onto various components of food rewardseeking behavior, gut microbiota ecosystem and molecular adaptation in both hypothalamic and mesolimbic structures. We used food choice paradigm associated with operant conditioning to lever press for food reward in order to dissect out how FOS supplementation could prevent or correct the consequence of chronic exposure to palatable, energy-rich diet onto the hedonic and motivational component of food seeking behavior. We manipulated the timing of FOS introduction in the diet using, first, a preventive-like approach in which animals were exposed to CTRL diet or HFHS diet with or without
FOS supplementation and second, a corrective-like approach in which animal were first raised on HFHS diet and then switched onto CTRL diet with or without FOS. In both cases, the consequences onto gut microbiota, hedonic and motivational aspect of food reward together with brain expression of genes involved homeostatic and non-homeostatic control of feeding.

We found that prebiotics act in synergy with the diet supplied to operate change in microbiota composition, tropism for palatable food, and hypothalamic and MCL response. Using targeted metagenomics approach, we could only identify selected changes in the gut-microbiota ecosystem, especially modifying the contents of Bifidobacterium spp., A. muciniphila- and Lactobacillus spp., but only in animals that were either raised or had been exposed chronically to HFHS diet (Figures 1I,J). In the same line we found that, while FOS addition to CTRL diet increased both the drive for palatable diet (Figures 2C-E) and hypothalamic expression of orexigenic neuropeptides NPY and AgRP (Figure 4B), prebiotic addition decreased the motivation to collect food rewards after a fast and decreased hypothalamic NPY content in HFHS fed animals (Figures 3G,I and 4B).

Surprisingly enough, in our hands FOS introduction to the diet only modestly affected HFHS-induced fat mass gain (Figure 1D; Figure S1B in Supplementary Material) but had no significantly impact of body weight gain or body weight loss after the transition from HFS to CTRL diet (Figures 1E-H; Figures S1C-E in Supplementary Material). However, despite the lack of effect on body weight, we could clearly demonstrate that the timing of prebiotic supplementation had a pivotal role in both molecular and behavioral responses in food reward seeking and consumption. After a 2-months HFHS exposure, we tested the capacity of prebiotic to revert molecular and behavioral dysfunctions induced by caloric overload. The shift onto CTRL diet similarly surfeited body weight gain regardless of FOS addition, however, in contrast to the chronic preventive approach, prebiotic supplementation resulted in decreased palatable food tropism and consumption (Figures 2F-I) without affecting operant performance (Figures $\mathbf{3 K}-\mathbf{Q}$ ) and was associated with concomitant increase in hypothalamic orexigenic markers and NAcc expression of gene involved in DA signaling. This latter result suggests that, unlike the preventive addition of FOS, prebiotic treatment after chronic HFHS exposure helped restoring the imbalance in MCL DA signaling and reward-driven tropism and overconsumption of palatable diet. Importantly, these changes primarily affected hedonic rather than motivational aspects of food reward and had a positive impact on food choice despite increased expression of hypothalamic orexigenic neuropeptides.

It is important to note that, while $10 \%$ FOS supplementation correlated with positive change in the gut microbiota ecosystem as expected from the literature, we did not observe a clear benefit on body weight. FOS introduction did mitigate fat mass gain in animal raised onto HFHS diet (Figure 1D) but on overall did not significantly modify body weight. Of note, however, while a decrease in body weight could be expected from prebiotic treatment it is important to highlight that while FOS supplementation has been shown to increase 
post-meal satiety and hunger, change in body weight were not always consistently observed. Indeed it when compared to other dietary fibers FOS supplementation was associated with body weight gain in lean rodents (28) and obese mice (49) while other report clearly show a preventive action of FOS on high-fat-mediated body weight gain (50). A genetic model of metabolic syndrome FOS supplementation was shown to drastically alleviate excessive feeding but had no impact on body weight (29). This study comes in addition with several report that clearly established the benefits of FOS onto glucose control and insulin sensitivity (51) and in that regards it is tempting to speculate that enhanced insulin sensitivity (29), while promoting a more healthy adipose development, could mitigate the overall body weight loss.

Despite overall similar body weight in within cohort, we found very different outcomes at the behavioral and molecular level when FOS supplementation was added during or after HFHS diet exposure. We first described a paradoxical action of FOS when assessed onto CTRL diet that increased the tropism for palatable diet when assessed on a two-food choice paradigm (Figures 2B-D) and while this result is in good agreement with the increase in hypothalamic orexigenic peptides (Figure 4B) it could also potentially be the consequence of anxiolytic-like properties of prebiotic (21) which might alleviate food neophobia classically observed in C57BL6 mice $(29,52)$ and result in faster maximization of palatable diet intake when given the choice. Importantly, however, it should be noted that our behavioral assay was designed to address how animals spontaneously prefer, or are willing to work for food reward and although alteration on reward feeding might lead to overconsumption (46), our protocol does not provide a measure on the long-term consequence onto body weight.

Chronic palatable diet exposure has been shown to promote changes in the reward system at both molecular and behavioral levels $(8,10,46)$. One possible explanation for this timing effect of prebiotic action that we observed might be encapsulated in the fact that pre exposure to energy-dense food might initiate both peripheral and central adaptive changes among which some could be selectively corrected by FOS addition. Indeed, once rodent have been exposed to reinforcing stimulus such as palatable diet or drug of abuse they are typically more prone to develop addictive-like behavior $(8,46)$. These adaptive changes can involve one or many components of the DA system $(7,8$, $46,53)$ in association with alteration of the gut microbial ecosystem (54). For instance, energy-dense food exposure leads to diet-induced central inflammation (55), neuropeptide signaling alteration $(29,44)$, and decrease in dopamine receptor abundance $(8,10,46)$, which would presumably participate in the development of addictive/compulsive eating behavior. Aside of a direct action onto the brain, energy-dense food also target the gut to control reward acquisition. Gut detection of dietary lipids have been shown to directly control DA release and action by route of the vagal nerves (56). These regulatory processes are probably part of larger integrative aspects by which the combination of diet and microbiota can influence host appetite through change in gut-derived metabolite, intestinal barrier, immune system (48).
Hence, the combination of HFHS exposure followed by prebiotic addition might overall change the microbia-gut-brain axis resulting in the fine-tuning or resetting of DA signaling and reward-driven behavior. Indeed, when FOS was added after HFHS exposure, we could observe a restoration of mesolimbic markers of DA signaling (Figures 4A,B) and, despite the increase in orexigenic NPY and AgRP observed the HFHS/CTRL-FOS group displayed strong reduction in food reward tropism (Figures 2F-H). This points at a rather dominant function of the reward system in the control of feeding in animals pre-exposed to palatable diet. Interestingly, the study from de Cossio and colleagues also described a beneficial action of prebiotic onto hyperphagia in obese animals that was independent of any changes in NPY, POMC was blunted by prebiotic addition, hypothalamic neuropeptide related (29).

Our results suggest that manipulating of the gut-brain axis can, in specific condition, exert a satietogenic effect primarily by modulating hedonic and motivational drive for food reward. This is in good agreement with the emerging concept that micriobiota-gut-brain axis is a potential avenue to modulate reward and in general addictive behavior $(57,58)$. Notwithstanding, a great limitation of our study lies in the use of targeted metagenomics approach that only accounted for specific bacterial strain changes. It is clear that prebiotic treatment will have consequences on gut flora that extend far beyond the changes that we described here (Figures 1I,J) and it is formally possible that one or multiple changes in the gut ecosystem that were not addressed here might reveal potential molecular underpinning by which bacterialhost interaction alters food reward.

In conclusion, our study depicts how timely controlled prebiotic manipulation can differentially and selectively affect positive reinforcement and motivational aspects of food rewardseeking behavior and demonstrate the efficacy of the gutmicrobiota-brain axis to operate molecular adaptations in neural substrates involved in both homeostatic and non-homeostatic control of body weight. However, further studies will be warrant to precisely describe the molecular underpinning of the bacterial-host interaction in the control of food reward.

\section{ETHICS STATEMENT}

All animal experiments were performed with approval of the Animal Care Committee of the University Paris Diderot-Paris 7 and according to European directives.

\section{AUTHOR CONTRIBUTIONS}

A-SD performed all the studies. JC, RD, CM, and MQ provided technical and conceptual support for behavioral and metabolic analysis and PCR analysis. AE and PC provide analysis of microbiota composition and conceptual support. FM and SL designed the study, secured the funding, and wrote the manuscript.

\section{ACKNOWLEDGMENTS}

This work was supported by a collaborative research grant from Laboratoire de Recherche Nutritionnelle KOT CEPRODI SA, 
Paris, the Centre National la Recherche Scientifique (CNRS), and the University Paris Diderot-Paris 7 . RD received a postdoctoral grant from the Région Île-de-France and a fellowship from the Région Ile de France and merit grant from the Société Francophone de Nutrition (SFN-LU). AE is research associate from the FRS-FNRS (Fonds de la Recherche Scientifique), PC is senior research associate from the FRS-FNRS. PC was the recipient of grants from FNRS. This work was supported by the FRFS-WELBIO under grant WELBIO-CGR-2017-C02 and the Funds Baillet Latour (Grant for Medical Research 2015). PC was a recipient of an ERC Starting Grant in 2013 (European Research Council, Starting grant 336452-ENIGMO). MQ is recipient of a Postdoctoral fellowship from Galician Government (Xunta de Galicia ED481B2014/039-0). We acknowledge the technical platform Functional and Physiological Exploration platform (FPE) of the Unit "Biologie Fonctionnelle et Adaptative,"

\section{REFERENCES}

1. Kahn BB, Flier JS. Obesity and insulin resistance. J Clin Invest (2000) 106:473-81. doi:10.1172/JCI10842

2. Kopelman PG, Hitman GA. Diabetes. Exploding type II. Lancet (1998) 352(Suppl 4):SIV5. doi:10.1016/S0140-6736(98)90267-7

3. Schwartz MW, Woods SC, Porte D Jr, Seeley RJ, Baskin DG. Central nervous system control of food intake. Nature (2000) 404:661-71. doi:10.1038/ 35007534

4. DiLeone RJ, Taylor JR, Picciotto MR. The drive to eat: comparisons and distinctions between mechanisms of food reward and drug addiction. Nat Neurosci (2012) 15:1330-5. doi:10.1038/nn.3202

5. Narayanan NS, Guarnieri DJ, DiLeone RJ. Metabolic hormones, dopamine circuits, and feeding. Front Neuroendocrinol (2010) 31:104-12. doi:10.1016/j. yfrne.2009.10.004

6. Berridge KC. 'Liking' and 'wanting' food rewards: brain substrates and roles in eating disorders. Physiol Behav (2009) 97:537-50. doi:10.1016/j. physbeh.2009.02.044

7. Wu C, Garamszegi SP, Xie X, Mash DC. Altered dopamine synaptic markers in postmortem brain of obese subjects. Front Hum Neurosci (2017) 11:386. doi:10.3389/fnhum.2017.00386

8. Johnson PM, Kenny PJ. Dopamine D2 receptors in addiction-like reward dysfunction and compulsive eating in obese rats. Nat Neurosci (2010) 13: 635-41. doi:10.1038/nn.2519

9. Denis RG, Joly-Amado A, Webber E, Langlet F, Schaeffer M, Padilla SL, et al. Palatability can drive feeding independent of AgRP neurons. Cell Metab (2015) 22:646-57. doi:10.1016/j.cmet.2015.07.011

10. Kenny PJ, Voren G, Johnson PM. Dopamine D2 receptors and striatopallidal transmission in addiction and obesity. Curr Opin Neurobiol (2013) 23:535-8. doi:10.1016/j.conb.2013.04.012

11. Berthoud HR. Mind versus metabolism in the control of food intake and energy balance. Physiol Behav (2004) 81:781-93. doi:10.1016/j.physbeh. 2004.04.034

12. Kelley AE, Baldo BA, Pratt WE. A proposed hypothalamic-thalamic-striatal axis for the integration of energy balance, arousal, and food reward. J Comp Neurol (2005) 493:72-85. doi:10.1002/cne.20769

13. Burokas A, Moloney RD, Dinan TG, Cryan JF. Microbiota regulation of the mammalian gut-brain axis. Adv Appl Microbiol (2015) 91:1-62. doi:10.1016/ bs.aambs.2015.02.001

14. Dinan TG, Cryan JF. Regulation of the stress response by the gut microbiota: implications for psychoneuroendocrinology. Psychoneuroendocrinology (2012) 37:1369-78. doi:10.1016/j.psyneuen.2012.03.007

15. Everard A, Belzer C, Geurts L, Ouwerkerk JP, Druart C, Bindels LB, et al. Cross-talk between Akkermansia muciniphila and intestinal epithelium controls diet-induced obesity. Proc Natl Acad Sci U S A (2013) 110:9066-71. doi:10.1073/pnas.1219451110
(University Paris Diderot, Sorbonne Paris Cité, BFA, UMR 8251 CNRS, F-75205 Paris, France) for metabolic and behavioral analysis. We also acknowledge the animal core facility "Buffon" of the University Paris Diderot-Paris 7/Institut Jacques Monod, Paris for animal husbandry and breeding. We thank Olja Kacanski for administrative support; Isabelle Le Parco, Ludovic Maingault, and Daniel Quintas for care of animals; Giuseppe Gangarossa, Claire Martin, and Chloé Berland for helpful comments on the manuscript; and Dr. Reginald Allouche for help in the experimental design.

\section{SUPPLEMENTARY MATERIAL}

The Supplementary Material for this article can be found online at https://www.frontiersin.org/articles/10.3389/fendo.2018.00273/ full\#supplementary-material.

16. Cani PD, Joly E, Horsmans Y, Delzenne NM. Oligofructose promotes satiety in healthy human: a pilot study. Eur J Clin Nutr (2006) 60:567-72. doi:10.1038/ sj.ejcn. 1602350

17. Cani PD, Neyrinck AM, Maton N, Delzenne NM. Oligofructose promotes satiety in rats fed a high-fat diet: involvement of glucagon-like Peptide-1. Obes Res (2005) 13:1000-7. doi:10.1038/oby.2005.117

18. Bindels LB, Neyrinck AM, Salazar N, Taminiau B, Druart C, Muccioli GG, et al. Non digestible oligosaccharides modulate the gut microbiota to control the development of leukemia and associated cachexia in mice. PLoS One (2015) 10:e0131009. doi:10.1371/journal.pone.0131009

19. Hadri Z, Chaumontet C, Fromentin G, Even PC, Darcel N, Bouras AD, et al. Long term ingestion of a preload containing fructo-oligosaccharide or guar gum decreases fat mass but not food intake in mice. Physiol Behav (2015) 147:198-204. doi:10.1016/j.physbeh.2015.04.039

20. Gibson GR, Hutkins R, Sanders ME, Prescott SL, Reimer RA, Salminen SJ, et al. Expert consensus document: The International Scientific Association for Probiotics and Prebiotics (ISAPP) consensus statement on the definition and scope of prebiotics. Nat Rev Gastroenterol Hepatol (2017) 14:491-502. doi:10.1038/nrgastro.2017.75

21. Burokas A, Arboleya S, Moloney RD, Peterson VL, Murphy K, Clarke G, et al. Targeting the microbiota-gut-brain axis: prebiotics have anxiolytic and antidepressant-like effects and reverse the impact of chronic stress in mice. Biol Psychiatry (2017) 82:472-87. doi:10.1016/j.biopsych.2016. 12.031

22. McLean PG, Bergonzelli GE, Collins SM, Bercik P. Targeting the microbiotagut-brain axis to modulate behavior: which bacterial strain will translate best to humans? Proc Natl Acad Sci U S A (2012) 109:E174; author reply E176. doi:10.1073/pnas.1118626109

23. Messaoudi M, Rozan P, Nejdi A, Hidalgo S, Desor D. Behavioural and cognitive effects of oligofructose-enriched inulin in rats. Br J Nutr (2005) 93(Suppl 1): S27-30. doi:10.1079/BJN20041348

24. Cansell C, Castel J, Denis RG, Rouch C, Delbes AS, Martinez S, et al. Dietary triglycerides act on mesolimbic structures to regulate the rewarding and motivational aspects of feeding. Mol Psychiatry (2014) 1095-105. doi:10.1038/mp.2014.31

25. Muccioli GG, Naslain D, Backhed F, Reigstad CS, Lambert DM, Delzenne NM, et al. The endocannabinoid system links gut microbiota to adipogenesis. Mol Syst Biol (2010) 6:392. doi:10.1038/msb.2010.46

26. Daubioul CA, Taper HS, De Wispelaere LD, Delzenne NM. Dietary oligofructose lessens hepatic steatosis, but does not prevent hypertriglyceridemia in obese zucker rats. J Nutr (2000) 130:1314-9. doi:10.1093/jn/ 130.5.1314

27. Cani PD, Possemiers S, Van de Wiele T, Guiot Y, Everard A, Rottier O, et al. Changes in gut microbiota control inflammation in obese mice through a mechanism involving GLP-2-driven improvement of gut permeability. Gut (2009) 58:1091-103. doi:10.1136/gut.2008.165886 
28. Li S, Yingyi G, Chen L, Lijuan G, Ou S, Peng X. Lean rats gained more body weight from a high-fructooligosaccharide diet. Food Funct (2015) 6:2315-21. doi:10.1039/c5fo00376h

29. de Cossio LF, Fourrier C, Sauvant J, Everard A, Capuron L, Cani PD, et al. Impact of prebiotics on metabolic and behavioral alterations in a mouse model of metabolic syndrome. Brain Behav Immun (2017) 64:33-49. doi:10.1016/j.bbi.2016.12.022

30. Cani PD, Lecourt E, Dewulf EM, Sohet FM, Pachikian BD, Naslain D, et al. Gut microbiota fermentation of prebiotics increases satietogenic and incretin gut peptide production with consequences for appetite sensation and glucose response after a meal. Am JClin Nutr (2009) 90:1236-43. doi:10.3945/ ajcn.2009.28095

31. Wang J, Tang H, Zhang C, Zhao Y, Derrien M, Rocher E, et al. Modulation of gut microbiota during probiotic-mediated attenuation of metabolic syndrome in high fat diet-fed mice. ISME J (2015) 9:1-15. doi:10.1038/ismej. 2014.99

32. Alcock J, Maley CC, Aktipis CA. Is eating behavior manipulated by the gastrointestinal microbiota? Evolutionary pressures and potential mechanisms. Bioessays (2014) 36:940-9. doi:10.1002/bies.201400071

33. Vucetic Z, Carlin JL, Totoki K, Reyes TM. Epigenetic dysregulation of the dopamine system in diet-induced obesity. J Neurochem (2012) 120:891-8. doi:10.1111/j.1471-4159.2012.07649.x

34. Kelley AE, Baldo BA, Pratt WE, Will MJ. Corticostriatal-hypothalamic circuitry and food motivation: integration of energy, action and reward. Physiol Behav (2005) 86:773-95. doi:10.1016/j.physbeh.2005.08.066

35. DiLeone RJ. The influence of leptin on the dopamine system and implications for ingestive behavior. Int J Obes (Lond) (2009) 33(Suppl 2):S25-9. doi:10.1038/ijo.2009.68

36. Berridge KC, Robinson TE. What is the role of dopamine in reward: hedonic impact, reward learning, or incentive salience? Brain Res Brain Res Rev (1998) 28:309-69. doi:10.1016/S0165-0173(98)00019-8

37. Davis JF, Tracy AL, Schurdak JD, Tschop MH, Lipton JW, Clegg DJ, et al. Exposure to elevated levels of dietary fat attenuates psychostimulant reward and mesolimbic dopamine turnover in the rat. Behav Neurosci (2008) 122: 1257-63. doi:10.1037/a0013111

38. Morton GJ, Cummings DE, Baskin DG, Barsh GS, Schwartz MW. Central nervous system control of food intake and body weight. Nature (2006) 443:289-95. doi:10.1038/nature05026

39. Wise RA. Role of brain dopamine in food reward and reinforcement. Philos Trans R Soc Lond B Biol Sci (2006) 361:1149-58. doi:10.1098/rstb. 2006.1854

40. Blouet C, Schwartz GJ. Hypothalamic nutrient sensing in the control of energy homeostasis. Behav Brain Res (2010) 209:1-12. doi:10.1016/j.bbr. 2009.12.024

41. Dorfman MD, Thaler JP. Hypothalamic inflammation and gliosis in obesity. Curr Opin Endocrinol Diabetes Obes (2015) 22:325-30. doi:10.1097/MED. 0000000000000182

42. Thaler JP, Yi CX, Schur EA, Guyenet SJ, Hwang BH, Dietrich MO, et al. Obesity is associated with hypothalamic injury in rodents and humans. J Clin Invest (2012) 122:153-62. doi:10.1172/JCI59660

43. Alsio J, Olszewski PK, Norback AH, Gunnarsson ZE, Levine AS, Pickering C, et al. Dopamine D1 receptor gene expression decreases in the nucleus accumbens upon long-term exposure to palatable food and differs depending on diet-induced obesity phenotype in rats. Neuroscience (2010) 171:779-87. doi:10.1016/j.neuroscience.2010.09.046

44. la Fleur SE, van Rozen AJ, Luijendijk MC, Groeneweg F, Adan RA. A freechoice high-fat high-sugar diet induces changes in arcuate neuropeptide expression that support hyperphagia. Int J Obes (Lond) (2010) 34:537-46. doi:10.1038/ijo.2009.257
45. van de Giessen E, la Fleur SE, de Bruin K, van den Brink W, Booij J. Freechoice and no-choice high-fat diets affect striatal dopamine D2/3 receptor availability, caloric intake, and adiposity. Obesity (Silver Spring) (2012) 20: 1738-40. doi:10.1038/oby.2012.17

46. Kenny PJ. Reward mechanisms in obesity: new insights and future directions. Neuron (2011) 69:664-79. doi:10.1016/j.neuron.2011.02.016

47. Saper CB, Chou TC, Elmquist JK. The need to feed: homeostatic and hedonic control of eating. Neuron (2002) 36:199-211. doi:10.1016/ S0896-6273(02)00969-8

48. van de Wouw M, Schellekens H, Dinan TG, Cryan JF. Microbiota-gut-brain axis: modulator of host metabolism and appetite. J Nutr (2017) 147:727-45. doi:10.3945/jn.116.240481

49. Liu TW, Cephas KD, Holscher HD, Kerr KR, Mangian HF, Tappenden KA, et al. Nondigestible fructans alter gastrointestinal barrier function, gene expression, histomorphology, and the microbiota profiles of diet-induced obese C57BL/6J mice. J Nutr (2016) 146:949-56. doi:10.3945/jn.115.227504

50. Nakamura $Y$, Natsume M, Yasuda A, Ishizaka M, Kawahata K, Koga J. Fructooligosaccharides suppress high-fat diet-induced fat accumulation in C57BL/6J mice. Biofactors (2017) 43:145-51. doi:10.1002/biof.147

51. Schneeberger M, Everard A, Gomez-Valades AG, Matamoros S, Ramirez S, Delzenne NM, et al. Akkermansia muciniphila inversely correlates with the onset of inflammation, altered adipose tissue metabolism and metabolic disorders during obesity in mice. Sci Rep (2015) 5:16643. doi:10.1038/ srep 16643

52. Kronenberger JP, Medioni J. Food neophobia in wild and laboratory mice (Mus musculus domesticus). Behav Processes (1985) 11:53-9. doi:10.1016/ 0376-6357(85)90102-0

53. Wang GJ, Volkow ND, Logan J, Pappas NR, Wong CT, Zhu W, et al. Brain dopamine and obesity. Lancet (2001) 357:354-7. doi:10.1016/S01406736(00)03643-6

54. Everard A, Geurts L, Caesar R, Van Hul M, Matamoros S, Duparc T, et al. Intestinal epithelial MyD88 is a sensor switching host metabolism towards obesity according to nutritional status. Nat Commun (2014) 5:5648. doi:10.1038/ ncomms 6648

55. Velloso LA, Araujo EP, de Souza CT. Diet-induced inflammation of the hypothalamus in obesity. Neuroimmunomodulation (2008) 15:189-93. doi:10.1159/ 000153423

56. Tellez LA, Medina S, Han W, Ferreira JG, Licona-Limon P, Ren X, et al. A gut lipid messenger links excess dietary fat to dopamine deficiency. Science (2013) 341:800-2. doi:10.1126/science.1239275

57. Skosnik PD, Cortes-Briones JA. Targeting the ecology within: the role of the gut-brain axis and human microbiota in drug addiction. Med Hypotheses (2016) 93:77-80. doi:10.1016/j.mehy.2016.05.021

58. Kiraly DD, Walker DM, Calipari ES, Labonte B, Issler O, Pena CJ, et al. Alterations of the host microbiome affect behavioral responses to cocaine. $S c i$ Rep (2016) 6:35455. doi:10.1038/srep35455

Conflict of Interest Statement: FM was employed by company KOT CEPRODI. The funders had no role in study design, data collection and analysis, decision to publish, or preparation of the manuscript. All authors declare no conflict of interest and competing interests.

Copyright $\odot 2018$ Delbès, Castel, Denis, Morel, Quiñones, Everard, Cani, Massiera and Luquet. This is an open-access article distributed under the terms of the Creative Commons Attribution License (CC BY). The use, distribution or reproduction in other forums is permitted, provided the original author(s) and the copyright owner are credited and that the original publication in this journal is cited, in accordance with accepted academic practice. No use, distribution or reproduction is permitted which does not comply with these terms. 